\title{
The detection of autoantibodies with an enzyme bridge method
}

\author{
P. M. FORD AND P. J. STOWARD \\ From the Departments of Medicine and Anatomy, University of Dundee
}

SYNOPSIS The horseradish peroxidase bridge method described by Mason, Phifer, Spicer, Swallow, $\stackrel{\leftrightarrow}{\stackrel{\leftrightarrow}{\circ}}$ and Dreskin (1969) has been found to be as sensitive and specific as fluorescence methods for detecting antinuclear factor and gastric parietal cell antibody, and to a lesser extent, antibodies to $\underset{N}{ }$ smooth muscle and mitochondria. However, the method has at least four practical advantages over $\stackrel{\sim}{\rightarrow}$ fluorescence techniques.

At present, tissue antigens are usually localized with antibodies tagged with either a fluorescent label or an enzyme such as horseradish peroxidase. Both tags have their disadvantages, most of which are avoided, however, in an enzyme bridge method introduced in 1969 by Mason et al. One purpose of this note is to report that the enzyme bridge method is, in some instances, as sensitive as fluorescence techniques and could usefully replace them in routine diagnostic work.

The bridge method involves the use of three antisera: (1) an antiserum to the antigen to be demonstrated; (2) an antiserum, from a different species, to the immunoglobulin component of (1); and (3) an antiserum from the same species as (1)

Received for publication 12 November 1973.

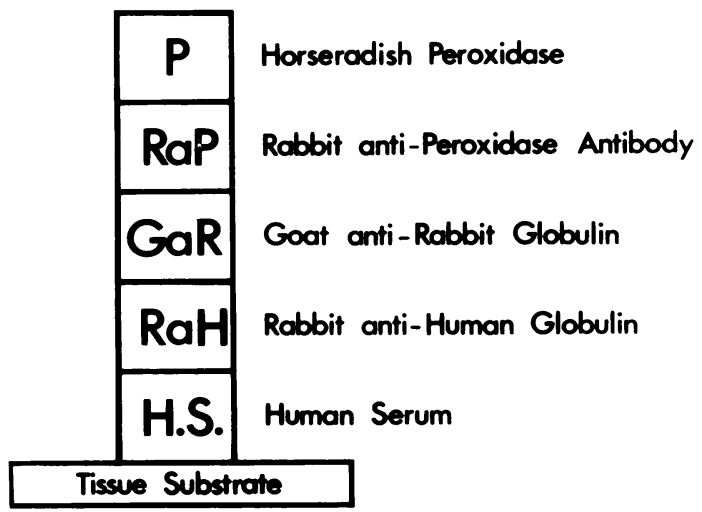

Fig 1 An example of an 'enzyme bridge' that can be used for detecting autoantibodies in a human serum. to a marker enzyme, in this case horseradish peroxi- 7 dase (fig 1). They are flooded onto a test substrate $\frac{0}{2}$ tissue in this order. Last of all the marker enzyme is rendered visible or electron opaque by an appropriate ${ }^{\gtrless}$ histochemical technique. If autoantibodies are $\vec{\varphi}$ present in the first antiserum, eg, human an nuclear factor, the appropriate antigen in the sutstrate tissue, eg, nuclei, is selectively staine Fortunately, non-organ-specific autoantibodies are not species specific and, therefore, rodent substrate tissue can be used for detecting human autoanti- $\$$ bodies by this test.

Until now, the bridge method has only been used for localizing lymphocyte surface antigens and a nephrotoxic antibody (Willingham, Spicer, and Graber, 1971; Choi and Good, 1972; Hoedemaeker, Feenstra, Nijkeuter, and Arends, 1972). Sternberger, 융 Hinton, Petrali, Meyer, and Cuculis (1971) sug- gested that the method could be used for estimating tissue antigens quantitatively but the preliminary 8 qualitative experiments of Bataillard and Campiche (1972) with ferritin do not seem to support this suggestion. Nevertheless, the method appears to have a bright future as shown by this work and also by the results reported here.

\section{Methods and Materials}

Human sera were obtained from patients with diseases of supposed immune aetiology and known

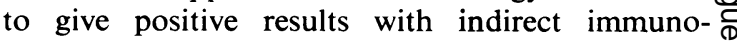
fluorescence tests. Staining patterns for several $\stackrel{\infty}{\rightarrow}$ antibodies were studied. These were antinuclear 0 factor and gastric parietal cell, smooth muscle and $\underset{P}{\stackrel{0}{ }}$ mitochondrial antibodies, five sera for each staining $\mathbb{D}$ pattern being studied. Sera giving negative results $\mathbb{D}$ 
with indirect immunofluorescence tests were used as controls. All sera were stored at $-25^{\circ} \mathrm{C}$ until use.

Cryostat sections, $5 \mu \mathrm{m}$ thick, of composite blocks of kidney, stomach, and liver, snap frozen on a liquid carbon dioxide expansion microtome chuck, of month-old rats were air-dried onto glass slides and used the same day.

Rabbit antihuman IgG and IgM and goat antirabbit globulin were obtained commercially (Hoechst Pharmaceuticals). They were diluted until they gave, with the bridge method, a specific localization of the antigen under investigation concomitant with a minimum background, ie, nonspecific staining.

Rabbit antihorseradish peroxidase serum was prepared by serial intramuscular injections, at two sites, of $4 \mathrm{mg}$ horseradish peroxidase (activity approximately 250 units $/ \mathrm{mg}$, purchased from Miles Laboratories) in $2 \mathrm{ml}$ Freund's complete adjuvant. A second dose was given about four weeks after the first. A booster injection was given about three days before withdrawing the sera. The antibody activity of the sera was found by immunoelectrophoresis to be in the IgG fraction. The antiserum was used either neat or as the IgG fraction prepared by ion exchange chromatography on QAE-Sephadex A-50.

\section{STAINING TECHNIQUE}

Substrate tissue sections were incubated with a 1:10 dilution of the serum under test, followed by rabbit antihuman immunoglobulin serum and goat antirabbit immunoglobulin serum in succession, both being used at their appropriate dilutions. Undiluted rabbit antihorseradish peroxidase serum was then applied, after which the sections were incubated for $30 \mathrm{~min}$ at room temperature in an aqueous solution of horseradish peroxidase $(1.25 \mathrm{mg} / 100 \mathrm{ml})$. The sections were incubated with each antiserum for $30 \mathrm{~min}$ at room temperature and washed for $15 \mathrm{~min}$ in $0.1 \mathrm{M}$ phosphate-buffered saline, $\mathrm{pH} 6.8$, between each application.

Peroxidase was localized by the method of Graham and Karnovsky (1966). Six drops of $3 \%$ hydrogen peroxide were added immediately before use to 10 $\mathrm{ml}$ of a saturated solution of 3,3'-diaminobenzidine (Sigma London Chemical Co.) in tris buffer, pH 7.6. Sections were incubated in this solution for four min, rinsed in distilled water and postfixed for three min in $2 \%$ osmium tetroxide. The preparations were then dehydrated through the ethanols and mounted in DPX.

\section{Results}

Satisfactory results were obtained with sera containing human antinuclear antibodies; nuclei in test

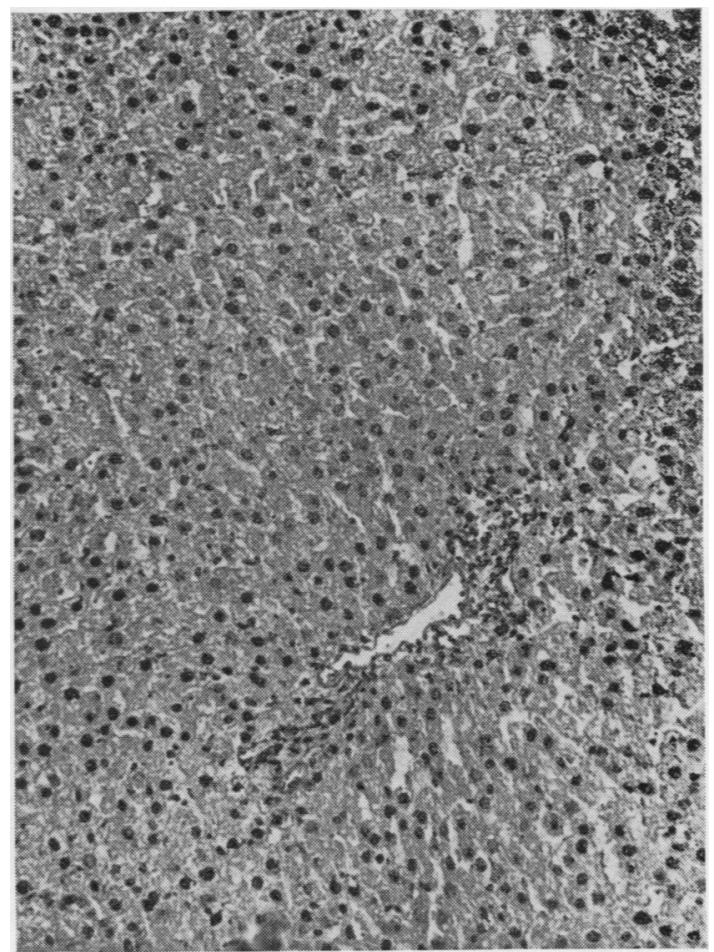

Fig 2 Antinuclear factor selectively localized on nuclei in liver by peroxidase bridge technique. $\times 130$.

tissues were sharply and clearly stained (fig 2 ). The immunofluorescence sandwich technique gave identical results when sera of equivalent titres were employed (see table), and no false positives were encountered when sera unreactive in the immunofluorescence technique were used.

Smooth muscle, eg, in the gastric mucosa, was also demonstrated satisfactorily with the enzyme

\begin{tabular}{lll}
\hline $\begin{array}{l}\text { Dilution Factor of } \\
\text { Antinuclear Antibody }\end{array}$ & \multicolumn{2}{l}{ Intensity of Staining or Fluorescence } \\
\cline { 2 - 3 } & Peroxidase & Immunofuorescence \\
\hline 10 & $3+$ & $3+$ \\
20 & $3+$ & $3+$ \\
40 & $2+$ & $2+$ \\
80 & $2+$ & $1+$ \\
160 & $1+$ & $1+$ \\
620 & \pm & \pm \\
\hline
\end{tabular}

Table Comparison of sensitivity of immunofluorescence and enzyme bridge methods in demonstrating antinuclear antibodies $^{1}$

${ }^{1}$ The intensity of specific staining or fluorescence was estimated subjectively on a scale in which $3+=$ very strong staining or intense fluorescence and $\pm=$ very weak or negligible staining or fluorescence. $\mathbf{0}=$ no staining or fluorescence. The same results were obtained with the two samples tested. 
bridge method. However, because of the unavoidable brown background staining, weak positives were probably missed.

Gastric parietal cells also stained well (fig 3) although some non-specific staining was obtained with control sera; this tended to make the recognition of weak positives difficult. Comparison with a control, however, usually overcame this difficulty (fig 4).

With mitochondrial antisera, test tissues were coloured brown overall but discrete granules were also evident. If a composite tissue block, including stomach, was used, the gastric parietal cells were clearly seen to be stained, thus making any diagnosis obvious.

The bridge method gave satisfactory results with both IgG and IgM rabbit antihuman sera. No difference was detected between the end-products when either whole rabbit antiperoxidase serum or its purified IgG fraction was used.

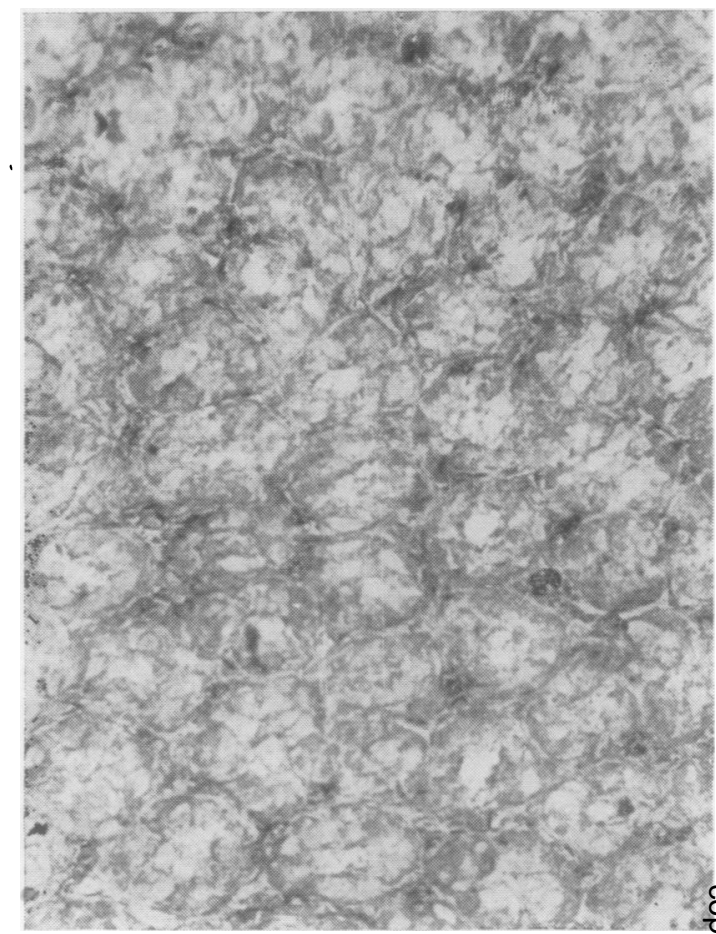

Fig 4

Fig 3 Gastric parietal cell antibody taken up selectively by parietal cells in a section of stomach. Compare with

Fig 4 Section of gastric mucosa treated with a control serum and subsequently subjected to the peroxidase bridge technique. Note the very weak and virtual absence of staining in gastric parietal cells compared with the staining shown in figure 3. A few mast cells exhibiting indigenous peroxidase activity are evident. $\times 330$.

\section{Discussion}

The immunoglobulin enzyme bridge method appears to be as sensitive and specific as immunofluorescence sandwich techniques, at least for detecting antinuclear factor and gastric parietal cell antibody. It was clearly not, however, more sensitive. This is disappointing as one would expect the bridge method, because it involves four globulin layers in contrast to the indirect immunofluorescent procedure's two, to be far more sensitive, although perhaps less specific. It is easy to speculate why the expected improvement in sensitivity did not materialize. For example, one could argue, without any supporting experimental evidence, that the histochemical technique for localizing peroxidase is inherently less sensitive than the detection of fluorescent dye labels. In our view such speculations are, for practical reasons, of little value.

To take one example, the sensitivity might be 
improved by using a purified serum, notwithstanding the fact that we found that a purified IgG fraction was not better than the crude serum. Although this fraction is labelled as pure, it still contains several non-specific components. A pure antibody, on the other hand, might substantially increase the sensitivity of the method more in line with expectations. But in the light of experience with immunofluorescence techniques, it may be undesirable in practice to use a pure antibody as the technique might then become so sensitive that it would be difficult, if not impossible, to eliminate 'background' staining.

In contrast to our observations, Sternberger (1972) found that using antisera of equivalent titre the peroxidase bridge method is 100 to 1000 times more sensitive than indirect immunofluorescence methods for identifying Treponema pallidum in spreads. However, in this situation 'background staining' does not arise and, therefore, Sternberger's observations are not strictly comparable with ours.

Although their sensitivity and specificity are similar, the enzyme bridge method has at least four advantages over fluorescent techniques. First, it gives a permanent preparation. Second, only a conventional light microscope is necessary for examining the 'stained' sections. Third, conjugation procedures are not required, thus avoiding the risks of denaturating sera and reducing their antigenicity. And last, a range of antihuman sera to different immunoglobulins may be used with just one anti- body, namely, antiperoxidase. On the other hand, the technique does have two disadvantages: compared with immunofluorescence techniques it requires more time to complete and the pale 'background' staining makes weak-positive smooth muscle and mitochondria antibodies more difficult to detect. Nevertheless, this does not detract from the value of the bridge technique for diagnostic work.

\section{References}

Bataillard, A. C., and Campiche, M. (1972). Ultrastructural localization of intracellular antigen by the IgG method. (Abstr.) Experientia (Basel), 28, 744.

Choi, Y. S., and Good, R. A. (1972). Development of chicken lymphoid system. II. Synthesis of primordial immunoglobulin M by the Bursa cells of chick embryo. J. exp. Med., 136, 8-20.

Graham, R. C., Jr., and Karnovsky, M. J. (1966). The early stages of absorption of injected horseradish peroxidase in the proximal tubules of mouse kidneys: ultrastructural cytochemistry by a new technique. J. Histochem. Cytochem., 14, 291-302.

Hoedemaeker, IP. J, Feenstra, K., Nijkeuter, A., and Arends, A. (1972). Ultrastructural localization of heterologous nephrotoxic antibody in the glomerular basement membrane of the rat. Lab. Invest., 26, 610-613.

Mason, T. E., Phifer, R. F., Spicer, S. S., Swallow, R. A., and Dreskin, R. B. (1969). An immunoglobulin-enzyme bridge method for localizing tissue antigens. J. Histochem. Cytochem., 17, 563569.

Sternberger, L. A. (1972). The unlabelled-antibody-peroxidase and the quantitative-immunouranium methods in light and electron immunohistochemistry. Tech. biochem. biophys. Morphol., 1, 67-68.

Sternberger, L. A., Hinton, D. M., Petrali, J. P., Meyer, H. G., and Cuculis, J. J. (1971). Approach to quantitative staining histochemistry. (Abstr.) J. Histochem. Cytochem., 19, 710.

Willingham, M. C., Spicer, S. S., and Graber, C. D. (1971). Immunocytologic labeling of calf and human lymphocyte surface antigens. Lab. Invest., 25, 211-219. 